\title{
Respiratory virus-related meningoencephalitis in adults
}

\author{
Seon-Jae Ahn ${ }^{1,2, *}$, Jangsup Moon ${ }^{2,3,}$, Jun-Sang Sunwoo ${ }^{1,4}$, Jin-Sun Jun ${ }^{5}$, Soon-Tae Lee ${ }^{2}$, Kyung-II Park ${ }^{2,6}$ \\ Keun-Hwa Jung ${ }^{2}$, Ki-Young Jung ${ }^{2}$, Manho Kim ${ }^{2,7,}$, Sang Kun Lee ${ }^{2,8}$, Kon Chu ${ }^{2}$ \\ ${ }^{1}$ Center for Hospital Medicine, Seoul National University Hospital, Seoul, Korea \\ ${ }^{2}$ Department of Neurology, Seoul National University Hospital, Seoul, Korea \\ ${ }^{3}$ Rare Disease Center, Seoul National University Hospital, Seoul, Korea \\ ${ }^{4}$ Department of Neurosurgery, Seoul National University Hospital, Seoul, Korea \\ ${ }^{5}$ Department of Neurology, Kangnam Sacred Heart Hospital, Hallym University College of Medicine, Seoul, Korea \\ ${ }^{6}$ Department of Neurology, Seoul National University Hospital Healthcare System Gangnam Center, Seoul, Korea \\ ${ }^{7}$ Protein Metabolism Medical Research Center, Seoul National University College of Medicine, Seoul, Korea \\ ${ }^{8}$ Neuroscience Research Institute, Seoul National University College of Medicine, Seoul, Korea
}

\section{Purpose}

Respiratory viruses (RVs) are pathogens that can cause central nervous system (CNS) infection, but previous research has been limited to a pediatric population. In recent years, several cases of adult RV meningoencephalitis have begun to be reported. We decided to research the CNS infection of RV in the entire neuroinfection registry.

\section{Methods}

We retrospectively reviewed the neurologic infection registry of Seoul National University (Seoul, Korea). Among a total of 661 patients in the registry, 10 adult patients were diagnosed with RV-related meningoencephalitis on RV multiplex polymerase chain reaction (PCR) screening test. We analyzed the clinical presentation, laboratory findings, and clinical course of the 10 patients.

\section{Results}

Three patients were definite RV meningoencephalitis who had positive PCR results from cerebrospinal fluid. The other seven patients were diagnosed with probable RV meningoencephalitis if they had positive PCR results in the sputum and negative results in other extensive workup.

\section{Conclusion}

RV-related meningoencephalitis should be considered a possible etiology in adult meningoencephalitis patients. To diagnose these viruses, screening test of RV PCR is recommended even in patients without upper respiratory infection symptoms.

Keywords: Central nervous system viral infections, Meningitis, Encephalitis, Polymerase chain reaction

\section{Introduction}

The respiratory virus (RV) group consists of viruses such as adenovirus; respiratory syncytial virus (RSV) A and B; rhinovirus A and B; coronavirus, influenza A and B; parainfluenza 1, 2, 3; and metapneumovirus. RVs mainly cause upper or lower respiratory tract infections, but they can also cause central nervous system (CNS) infection, mostly in children [1]. Adult cases of RV-related meningoencephalitis have been reported in a limited number of patients, predominantly those with influenza virus [2]. How-

Received: November 8, 2020 Revised: November 14, 2020 Accepted: November 23, 2020

Correspondence: Kon Chu

Department of Neurology, Seoul National University Hospital, 101 Daehak-ro, Jongno-gu, Seoul 03080, Korea

E-mail: stemcell.snu@gmail.com

ORCID: https://orcid.org/0000-0001-5863-0302

*These authors contributed equally to this study as co-first authors.

Copyright ( 2021 by The Korean Encephalitis and Neuroinflammation Society

This is an open access article distributed under the terms of the Creative Commons Attribution Non-Commercial License (http://creativecommons.org/licenses/by-nc/4.0/) which permits unrestricted non-commercial use, distribution, and reproduction in any medium, provided the original work is properly cited. 
ever, up to $66 \%$ of adult encephalitis cases fail to have a causative pathogen identified despite extensive diagnostic laboratory tests [3]. Therefore, further research is needed to identify unusual but detectable pathogens such as RV. We retrospectively reviewed the neurological infection registry, and analyzed the pathogenic etiology of the CNS infections, including the RVs.

\section{Methods}

From March 2012 to December 2015, patients visiting Seoul National University Hospital (Seoul, Korea) with a clinical suspicion of CNS infection were enrolled in the neurological infection registry of Seoul National University Hospital (Seoul, Korea). The inclusion criteria were as follows; (1) clinical suspicion of CNS infection, (2) $\geq 18$ years of age, and (3) cerebrospinal fluid (CSF) pleocytosis. The exclusion criteria were as follows; (1) CNS infection related to a recent surgical intervention, or (2) the presence of an additional etiology, such as autoimmune encephalitis or metabolic encephalopathy.

All patients in this registry underwent RV multiplex polymerase chain reaction (PCR) (Anyplex II RV 16 Detection; Seegene Inc., Seoul, Korea) analysis of CSF and sputum. This test provides qualitative analysis for 16 species of RV (adenovirus, influenza A and $\mathrm{B}$, parainfluenza virus $1 / 2 / 3 / 4$, rhinovirus $\mathrm{A} / \mathrm{B} / \mathrm{C}$, RSV A and $\mathrm{B}$, bocavirus $1 / 2 / 3 / 4$, metapneumovirus, coronavirus $229 \mathrm{E}$, coronavirus NL63, coronavirus OC43, and enterovirus). Other extensive diagnostic tests were performed, which included CSF culture, CSF PCR, and serum antibody tests for bacteria and viruses. Work-ups for rare pathogens such as tuberculosis, fungus, and parasites were also performed. A clinical diagnosis of meningitis or encephalitis was made based on the initial manifestation of symptoms. Meningitis was characterized by fever and headache without any neurological symptoms. Encephalitis was characterized by headache, altered level of consciousness, and symptoms and signs of cerebral dysfunction such as cognitive impairment, behavioral changes, focal neurologic abnormalities, and seizures [4]. Individual patients were treated according to clinical decisions made by an expert physician from the neurological infection department. Because of its retrospective nature, this study was exempted from the approval of the Institutional Review Board and the written informed consent of the subject.

\section{Results}

A total of 661 patients were diagnosed with CNS infection and enrolled in the registry. Among these 661 CNS infection pa- tients, 351 had meningitis, 275 encephalitis, and 35 brain abscess. Etiological pathogens were confirmed in 421 patients $(63.7 \%)$. Viral infection $(253,60.1 \%)$ was the major etiology in pathogen-confirmed CNS infection, followed by bacterial infection (105, 24.9\%), mycobacterial infection (25, 5.9\%) and fungal infection (21, 5.0\%). In pathogen-confirmed viral infection, EBV was the leading etiology $(73,28.9 \%)$ followed by herpes simplex virus (HSV; 62, 24.5\%), varicella-zoster virus (VZV; 53, 20.9\%), and enterovirus $(51,20.2 \%)$.

A total of 10 patients were diagnosed with RV-related meningoencephalitis. RV PCR of CSF or sputum yielded positive results, with no other findings on extensive workup. The median age of the patients was 38 years (range, 21-72 years). Four patients were clinically diagnosed with encephalitis and the others were diagnosed with meningitis. Six patients $(60.0 \%)$ had prior upper respiratory infection (URI) symptoms before manifestation of CNS infection. Leptomeningeal enhancement was the most frequent finding $(70.0 \%)$ observed on brain magnetic resonance imaging (MRI). Antiviral therapy was administered in 3 of 4 encephalitis patients and 2 of 7 meningitis patients. All of the patients recovered fully without any neurological sequelae, except for one patient (patient 3) who deteriorated despite antiviral treatment (Table 1).

We classified these patients into two different groups according to PCR results. The first was the "definite" group, in which RV presence was confirmed by CSF PCR, and the second was the "probable" group, in which RV presence could be confirmed only by sputum PCR.

\section{Definite respiratory virus meningoencephalitis}

Three patients were classified into the "definite" group. Two patients had positive PCR results for influenza A in CSF. The other patient had positive results for human parainfluenza 3 virus in CSF.

Patient 1 was a 40 -year-old female. She visited the emergency room for altered mentality and fever lasting 5 days. Before the onset of neurologic symptoms, she complained of cough, rhinorrhea, and mild fever. Initial CSF revealed pleocytosis with a white blood cell (WBC) count of $129 / \mathrm{mm}^{3}$ (polymorphonucleocytes, $82 \%$; lymphocytes, $4 \%$; other cells, $14 \%$ ), and elevated protein (153 mg/dL). Initial brain MRI showed leptomeningeal enhancement, but electroencephalography (EEG) did not demonstrate abnormal findings. On extensive diagnostic workup, CSF PCR was positive for influenza A virus. She was treated with oseltamivir and recovered fully after 2 weeks of antiviral therapy; she was discharged at a modified Rankin Scale (mRS) 


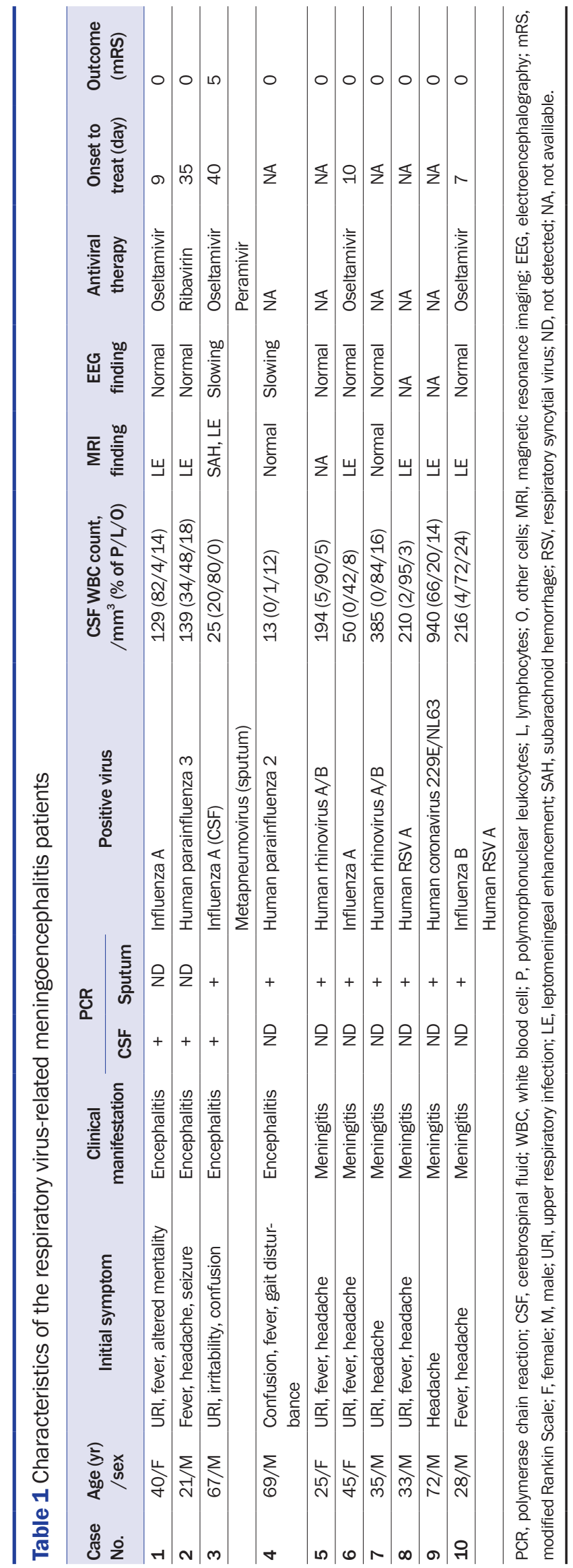

score of 0.

Patient 2 was a 21-year-old male who presented with first-onset seizure. He had a generalized tonic-clonic seizure after complaining of headache for 5 days. Initial CSF showed pleocytosis with red blood cells (RBC) of $12 / \mathrm{mm}^{3}$, a WBC of $139 / \mathrm{mm}^{3}$ (polymorphonucleocytes, 34\%; lymphocytes, $48 \%$; other cells, $18 \%$ ), and elevated protein $(52 \mathrm{mg} / \mathrm{dL})$. RV PCR of CSF was positive for human parainfluenza virus 3; otherwise, there were no abnormal findings on extensive diagnostic workup. Brain MRI showed leptomeningeal enhancement and EEG demonstrated no focal epileptiform discharge but did reveal generalized intermittent rhythmic delta activity. He was treated with ribavirin for 1 week, after which he was clinically recovered and did not complain of any headache or seizure. Follow-up EEG was also normal after ribavirin treatment.

Patient 3 was a 67 -year-old male who had been hospitalized previously for headache, right-side weakness, and transcortical motor aphasia. Prior to headache, he suffered from cough and rhinorrhea for 1 month. Brain computed tomography at a previous hospital showed cortical subarachnoid hemorrhage (SAH). He was treated with corticosteroids in order to reduce intracranial pressure. Initial CSF results revealed pleocytosis with RBC of $270 / \mathrm{mm}^{3}$, WBC of $25 / \mathrm{mm}^{3}$ (lymphocytes, $80 \%$; polymorphonucleocytes, $20 \%)$, and elevated protein $(214.7 \mathrm{mg} / \mathrm{dL})$. He recovered partially and was discharged after 1 week of steroid treatment. However, before long, he started to show confused mentality again and was transferred to our hospital for a second opinion. Follow-up CSF analysis in our hospital revealed persistent pleocytosis with an RBC of $18 / \mathrm{mm}^{3}$ and WBC of $16 / \mathrm{mm}^{3}$ (lymphocytes, 13; other cells, 3), and elevated protein (137 mg/ $\mathrm{dL}$ ). On RV PCR, the CSF specimen was positive for influenza A and the sputum specimen was positive for human metapneumovirus. On brain MRI, cortical SAH (Figure 1A) was aggravated and leptomeningeal enhancement (Figure 1B) was observed. Initially, acyclovir was administered for 1 week but was changed to oseltamivir after CSF PCR results were obtained. Although we treated him with oseltamivir for 2 weeks and follow-up CSF PCR results were negative, his mental status worsened. Finally, we administered intravenous peramivir, but the patient entered a persistent vegetative state (mRS score 5 ) without clinical response.

\section{Probable respiratory virus meningoencephalitis} We classified the other seven patients into the clinically "probable" group. Patients in this group were clinically diagnosed with meningoencephalitis and no pathogens were found on extensive workup other than positive RV PCR of sputum. There were 
Figure 1 Brain MRI of a respiratory virus-related encephalitis patient with a poor outcome (patient 3)
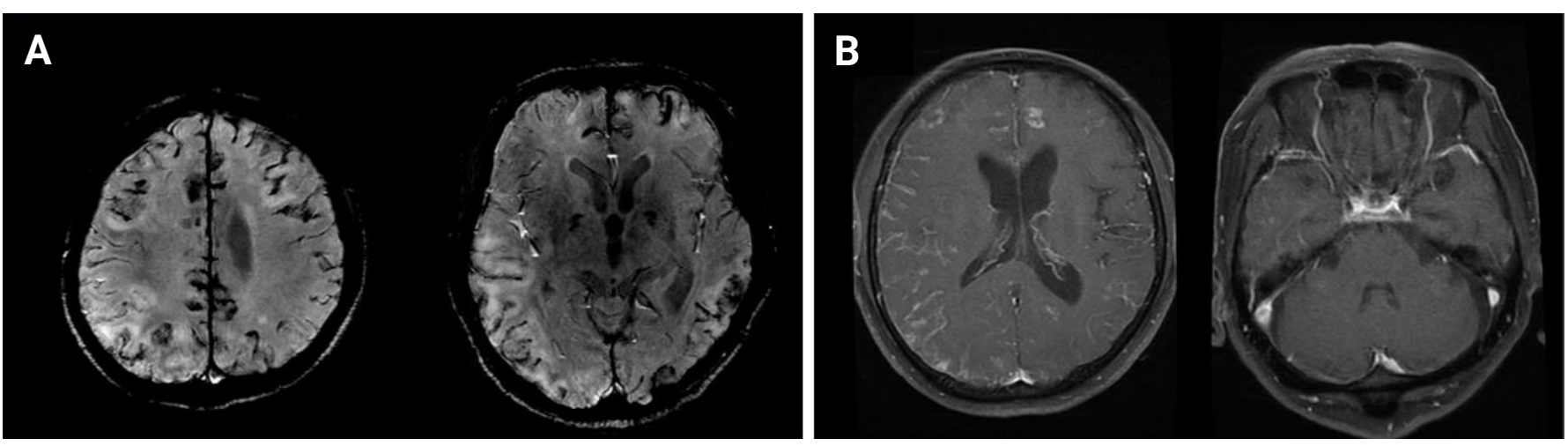

(A) Diffuse cortical subarachnoid hemorrhage on susceptibility-weighted angiography MRI. (B) Leptomeningeal enhancement on T1-weighted contrastenhanced MRI

MRI, magnetic resonance imaging.

two human rhinovirus A/B infections, one of influenza A, one of human parainfluenza virus 2 , one human coronavirus $229 \mathrm{E}$ / NL63, and one case with both influenza B and human RSV A. Four of these patients had URI symptoms prior to headache. Two patients whose PCR results were positive for influenza A were treated with oseltamivir, and the other patients underwent conservative management. Four patients exhibited leptomeningeal enhancement on MRI. Specific clinical information is presented in Table 1.

\section{Discussion}

Here, we demonstrated that, although rare, RVs can be a causative pathogen of meningoencephalitis in adults. According to our data from an adult population, $1.5 \%$ of total CNS infection patients were diagnosed with RV-related meningoencephalitis. This accounts for $4.0 \%$ of the total CNS viral infections.

Recently, neurologic manifestations of respiratory viral infection have come to the fore. RVs can invade the CNS through either a hematogenous route or a peripheral nerve route [5]. Many cases of RV-associated meningoencephalitis have been reported in adult patients with adenovirus [6], bocavirus [7], influenza A [810], influenza B [11,12], parainfluenza [13], and metapneumovirus [14-17]. Reports of meningoencephalitis caused by diverse types of RVs are more common in pediatric cases. RV-related encephalitis comprises approximately $20 \%$ of all encephalitis cases in children aged 1 month to 15 years [2]. However, even in adults, RVs remain an important pathogenic cause of meningoencephalitis around the world.
The diagnostic strategy for RV-related meningoencephalitis is similar to other infectious meningoencephalitis. PCR and real time-PCR assays of CSF for the detection of viruses are the most reliable diagnostic tools. A wide range of PCR tests should be carried out, including RV panel, HSV-1, HSV-2, VZV, CMV, and enterovirus, among others. Serologic tests, including serum and CSF specimens, are also helpful to specific etiological diagnosis. In the case of respiratory viral infection, we believe PCR testing of sputum will help establish a diagnosis [18].

Detailed history taking about prodromal symptoms, recent travel, geographic location, exposure history, and occupation provides important clinical clues regarding infectious pathogens. Nevertheless, clinical symptoms and neurologic examination findings are similar among CNS infections. In our study, it is remarkable that a considerable portion of RV-related meningoencephalitis patients ( 4 of 10) did not have URI symptoms. In 2009, a prospective longitudinal study showed that respiratory pathogens are frequently detected in samples not only from children with respiratory symptoms (56\%) but also from those without respiratory symptoms (40\%) [19]. Therefore, we believe that a respiratory PCR panel should be routinely conducted in patients with suspected CNS infections.

Most RV-related meningoencephalitis patients showed favorable outcomes. RV-related encephalitis is treatable with properly timed antiviral therapy. In particular, influenza A encephalitis can be treated with antiviral therapy [20,21]. Influenza A encephalitis can be fatal not only in children but also in adults [2224]. Cortical SAH can develop from influenza A infection [25,26]. In our cases, patient 3 showed a poor clinical outcome despite 
antiviral treatment. He already had a cortical SAH long before antiviral treatment began. The delay between onset and administration of the antiviral agent could be the reason why he was the only patient with clinical deterioration. This case showed the importance of fast and accurate diagnosis of RV-related meningoencephalitis [27]. Ribavirin, a broad-spectrum antiviral agent, is used to treat Paramyxoviridae pneumonia, such as human RSV, parainfluenza virus, and metapneumovirus [28]. Intravenous ribavirin was effective in Nipha-virus encephalitis [29], which is caused by another Paramyxoviridae disease. In our study, patient 2 was also successfully treated with ribavirin. Ribavirin is the treatment of choice when any patient is infected with RVs like those described above.

Our study has some limitations in the certainty of diagnosis. Because of the low detectability of viral nucleic acids in CSF, many cases of encephalitis were diagnosed based on the results of serological tests, antigen detection, viral culture, and nucleic acid detection from sputum, stool, urine, or blood [4]. Positive PCR results from sputum also can be indirect evidence of a diagnosis. Previous studies of RV-related meningoencephalitis and other studies of CNS infection etiology have used these indirect methods for specific etiological diagnosis [3]. Nevertheless, these indirect tools provide a lower level of confidence than PCR results from CSF and have the possibility of detecting asymptomatic coinfections limited to the upper respiratory system. This is an unavoidable limitation of our study. Therefore, we chose to divide the patients into two groups based on the certainty of diagnosis.

In conclusion, this is the first etiological study of adult RV-related meningoencephalitis in a large CNS infection registry. Clinicians should keep in mind that, although rare, RVs can cause acute meningoencephalitis in adult patients, even in those without URI symptoms.

\section{Conflicts of Interest}

Jangsup Moon, Soon-Tae Lee, Kyung-Il Park, Keun-Hwa Jung, Sang Kun Lee, Kon Chu have been editorial board of encephalitis since October 2020. They were not involved in the review process of this original article. No other potential conflict of interest relevant to this article was reported.

\section{Author Contributions}

Conceptualization: ST Lee, K Chu; Data curation: SJ Ahn; Formal analysis: SJ Ahn, J Moon; Funding acquisition: M Kim;
Methodology: SJ Ahn, K Chu; Visualization: SJ Ahn, JS Jun; Supervision: J Sunwoo, ST Lee, KI Park, KH Jung, KY Jung, M Kim, SK Lee, K Chu; Validation: KI Park, KH Jung, KY Jung, M Kim, SK Lee; Writing-original draft: SJ Ahn, J Moon; Writing-review \& editing: SJ Ahn, J Moon, J Sunwoo, JS Jun, K Chu.

\section{Acknowledgements}

This work was supported by the Brain Research Program through the National Research Foundation of Korea (NRF) funded by the Ministry of Science, ICT \& Future Planning (NRF-2016M33 C7A1914002).

\section{References}

1. Koskiniemi M, Korppi M, Mustonen K, et al. Epidemiology of encephalitis in children. A prospective multicentre study. Eur J Pediatr 1997; 156:541-545.

2. Koskiniemi M, Rantalaiho T, Piiparinen H, et al. Infections of the central nervous system of suspected viral origin: a collaborative study from Finland. J Neurovirol 2001;7:400-408.

3. Kupila L, Vuorinen T, Vainionpää R, Hukkanen V, Marttila RJ, Kotilainen P. Etiology of aseptic meningitis and encephalitis in an adult population. Neurology 2006;66:75-80.

4. Steiner I, Budka H, Chaudhuri A, et al. Viral encephalitis: a review of diagnostic methods and guidelines for management. Eur J Neurol 2005;12:331-343.

5. Bohmwald K, Gálvez NMS, Ríos M, Kalergis AM. Neurologic alterations due to respiratory virus infections. Front Cell Neurosci 2018; 12:386.

6. Fianchi L, Scardocci A, Cattani P, Tartaglione T, Pagano L. Adenovirus meningoencephalitis in a patient with large B-cell lymphoma. Ann Hematol 2003;82:313-315.

7. Mori D, Ranawaka U, Yamada K, et al. Human bocavirus in patients with encephalitis, Sri Lanka, 2009-2010. Emerg Infect Dis 2013; 19:1859-1862.

8. Santini M, Kutleša M, Zarković K, Draženović V, Barsic B. Influenza A $2009 \mathrm{H} 1 \mathrm{N1}$ encephalitis in adults with viral RNA in cerebrospinal fluid. Scand J Infect Dis 2012;44:992-996.

9. Cunha BA, Fear GL, Chawla K. A rare case of influenza A in a hospitalized adult presenting with encephalitis and a seizure. IDCases 2018; 12:153-155.

10. Midha D, Kumar A, Vasudev P, Iqbal ZA, Mandal AK. Adult influenza A (H1N1) related encephalitis: a case report. Indian J Crit Care Med 2018;22:384-387.

11. McCullers JA, Facchini S, Chesney PJ, Webster RG. Influenza B virus encephalitis. Clin Infect Dis 1999;28:898-900. 
12. Ak Ö, Biteker F, Cag Y, et al. Influenza B-associated encephalopathy in two adults. J Infect Chemother 2012;18:961-964.

13. Craver RD, Gohd RS, Sundin DR, Hierholzer JC. Isolation of parainfluenza virus type 3 from cerebrospinal fluid associated with aseptic meningitis. Am J Clin Pathol 1993;99:705-707.

14. Tan YL, Wee TC. Adult human metapneumovirus encephalitis: a case report highlighting challenges in clinical management and functional outcome. Med J Malaysia 2017;72:372-373.

15. Jeannet N, van den Hoogen BG, Schefold JC, Suter-Riniker F, Sommerstein R. Cerebrospinal fluid findings in an adult with human metapneumovirus-associated encephalitis. Emerg Infect Dis 2017; 23:370.

16. Fok A, Mateevici C, Lin B, Chandra RV, Chong VH. Encephalitis-associated human metapneumovirus pneumonia in adult, Australia. Emerg Infect Dis 2015;21:2074-2076.

17. Sánchez Fernández I, Rebollo Polo M, Muñoz-Almagro C, et al. Human metapneumovirus in the cerebrospinal fluid of a patient with acute encephalitis. Arch Neurol 2012;69:649-652.

18. Tyler KL. Acute viral encephalitis. N Engl J Med 2018;379:557-566.

19. van der Zalm MM, van Ewijk BE, Wilbrink B, Uiterwaal CS, Wolfs TF, van der Ent CK. Respiratory pathogens in children with and without respiratory symptoms. J Pediatr 2009;154:396-400.

20. Fox J, Barbour S, Junco SJ. Central nervous system laboratory-confirmed influenza meningo-encephalitis treated with peramivir. Int J Antimicrob Agents 2018;52:517-518.

21. Alsolami A, Shiley K. Successful treatment of influenza-associated acute necrotizing encephalitis in an adult using high-dose oseltamivir and methylprednisolone: case report and literature review. Open Forum Infect Dis 2017;4:ofx145.

22. Simon M, Hernu R, Cour M, Casalegno JS, Lina B, Argaud L. Fatal influenza A(H1N1)pdm09 encephalopathy in immunocompetent man. Emerg Infect Dis 2013;19:1005-1007.

23. Akins PT, Belko J, Uyeki TM, Axelrod Y, Lee KK, Silverthorn J. H1N1 encephalitis with malignant edema and review of neurologic complications from influenza. Neurocrit Care 2010;13:396-406.

24. Fugate JE, Lam EM, Rabinstein AA, Wijdicks EF. Acute hemorrhagic leukoencephalitis and hypoxic brain injury associated with H1N1 influenza. Arch Neurol 2010;67:756-758.

25. AbdelRazek MA, Leone MJ, Venna N. Hemorrhagic encephalitis associated with H3N2 influenza A viral pneumonia. Neurology 2018; 90:336-337.

26. Takeda H, Isono M, Kobayashi H. Possible acute hemorrhagic leukoencephalitis manifesting as intracerebral hemorrhage on computed tomography: case report. Neurol Med Chir (Tokyo) 2002;42:361-363.

27. Meijer WJ, Linn FH, Wensing AM, et al. Acute influenza virus-associated encephalitis and encephalopathy in adults: a challenging diagnosis. JMM Case Rep 2016;3:e005076.

28. Abed Y, Boivin G. Treatment of respiratory virus infections. Antiviral Res 2006;70:1-16.

29. Chong HT, Kamarulzaman A, Tan CT, et al. Treatment of acute Nipah encephalitis with ribavirin. Ann Neurol 2001;49:810-813. 